\title{
Comparison of two functional kappa light-chain transcripts amplified from a hybridoma
}

\author{
Juan Yang ${ }^{1}$ \\ Huifen $\mathrm{Zhu}^{1}$ \\ Zheng $\operatorname{Tan}^{1}$ \\ Fengrong $\mathrm{He}^{1}$ \\ Xiaoxu Sun ${ }^{1}$ \\ Yi Hong ${ }^{1}$ \\ Heyu $\mathrm{Hu}^{1}$ \\ Jing Bian ${ }^{1}$ \\ Yu Lin ${ }^{2}$ \\ Ping Lei ${ }^{1 *}$ \\ Guanxin Shen ${ }^{1}$
}

\author{
${ }^{1}$ Department of Immunology, Tongji Medical College, Huazhong University \\ of Science and Technology, Wuhan, People's Republic of China \\ ${ }^{2}$ Unit for Laboratory Animal Medicine, Department of Microbiology and \\ Immunology, and Center for Computational Medicine and Bioinformatics, \\ University of Michigan Medical School, Ann Arbor, MI, USA
}

\section{Abstract}

Three heavy-chain and three kappa $(\kappa)$-chain transcripts were amplified from hybridoma cells secreting a monoclonal antibody $(m A b)$ against transferrin receptor. Sequence analysis via IMGT/V-QUEST yielded the functional/aberrant prediction. Two functional $\kappa$-chain transcripts, $\mathrm{V}_{\kappa} 2$ and $\mathrm{V}_{\kappa} 3$, and one functional $\mathrm{VH} 1$ were revealed. Comprehensive bioinformatics analyses including sequence alignment, phylogenetic tree, somatic hypermutation prediction, and three-dimensional-molecular structure modeling were used to predict the origin of the two $\kappa$-chain transcripts. The results of bioinformatics analysis suggest that

Keywords: antibody engineering, chimeric antibody, transferrin receptor, sequence analysis, hybridoma, molecular modeling

\section{Introduction}

The transferrin receptor (TfR/CD71) is a cell-membraneassociated glycoprotein involved in cellular uptake of iron and

Abbreviations: $\mathrm{CHO}$, Chinese hamster ovary cells; $\mathrm{CDR}$, complementary determining region; Fab, fragment, antigen binding region; FCM, flow cytometry; FR, framework region; $m A b$, monoclonal antibody; scFv, single chain antibody fragment; TM, template modeling score; TfR/CD71, transferrin receptor; $V$, variable regions; $V H$, heavy chain variable region; $V L$, light chain variable region; $3 D$, three-dimensional.

*Address for correspondence: Ping Lei, PhD, Associate Professor, Department of Immunology, Tongji Medical College, Huazhong University of Science and Technology, Wuhan 430030, People's Republic of China. Tel.: +86 2783692611 ; Fax: +86 27 83693500; e-mail:

shenguanxin@mail.hust.edu.cn or adaleip@sina.com.

Received 8 October 2012; accepted 14 December 2012

DOI: 10.1002/bab.1080

Published online 30 April 2013 in Wiley Online Library

(wileyonlinelibrary.com)
$\mathrm{V}_{\kappa} 3$ is derived from the myeloma partner of the hybridoma; $\mathrm{V}_{\kappa} 2$ is derived from B-cell. Functional transcripts $\mathrm{V} H 1$ and $\mathrm{V}_{\kappa} 2$ and $\mathrm{V}_{\kappa} 3$ were then used to construct two chimeric antibodies chi-C2 $\left(\mathrm{V}_{\kappa} 2-\mathrm{V} H 1\right)$ and chi-C3 $\left(\mathrm{V}_{\kappa} 3-\mathrm{VH} 1\right)$, respectively. Antigen-binding experiments showed that only chi-C2 remained the same affinity as its parental mAb. Possible explanations for the coexistence of two functional $\kappa$-chain transcripts and the different affinity of the two chimeric antibodies are discussed. (c) 2013 International Union of Biochemistry and Molecular Biology, Inc. Volume 60, Number 3, Pages 289-297, 2013 in regulation of cell growth. Additionally, it is a selective target for cancer therapy [1-4]. We previously reported that a murine monoclonal anti-human TfR antibody showed excellent antitumor activity in vitro $[5,6]$. However, because of the murine monoclonal antibodies' (mAbs) immunogenicity and poor ability to recruit immune effector mechanisms, the feasibility of applying them in the clinic is limited. An attempt to overcome these obstacles is to generate human-mouse chimeric mAbs.

A prerequisite for the use of recombinant antibody technology from hybridomas or immune repertoires requires reliable cloning of functional immunoglobulin genes. Amplifying specific variable (V) regions of any given immunoglobulin is challenging. A major problem is obtaining sequence information about mAbs. The problem stems from the occurrence of aberrant mRNAs, which are transcribed from rearranged, but nonfunctional, heavy- and light-chain genes in the hybridoma [7]. It is known that immunoglobulin genes are highly diverse in their nucleotide sequences, as is the antibody repertoire they encode. Genes encoding the $\mathrm{V}$ region of immunoglobulin 
TABLE 1

PCR primers used for amplification of $\mathrm{Ig}$ variable regions

Primer

Primer sequence $\left(5^{\prime}-3^{\prime}\right)$

VL-f 1

VL-f 2

VL-f 3

VL-f 4

VL-f 5

VL-r 1

$\mathrm{VH}-\mathrm{f} 1$

$\mathrm{VH}-\mathrm{f} 2$

$\mathrm{VH}-\mathrm{f} 3$

VH-r 1
GGGGTCGACCTCACCATGGAGACAGACACACTCCTGCTAT

GGGGTCGACCTCACCATGGATTTTCAAGTGCAGATTTTCAG

GGGGTCGACCTCACCATGGAG[A/T]CACA[G/T][A/T]CTCAGGTCTTT[G/A]TA

GGGGTCGACCTCACCATG[G/T]CCCC[A/T][A/G]CTCAG[C/T]T[C/T]CTT/G]GT

GGGGTCGACCTCACCATGAAGTTGCCTGTTAGGCTGTTG

GCGGAATTCAGCCCGTTT[G/T]ATTTCCA[A/G]CTT

GGGGTCGACCTCACCATGG[A/G]ATG[C/G]AGCTG[T/G]GT[C/A]AT[C/G] CTCTT GGGGTCGACCTCACCATG[A/G]ACTTCGGG[T/C]TGAGCT[T/G]GGTTTT

GGGGTCGACCTCACCATGGCTGTCTTGGGGCTGCTCTTCT

GGAAGATCTATAGACAGATGGGGGTGTCGTTTTGGC

VL-f, VL forward; VL-r, VL reverse; VH-f, VH forward; VH-r, VH reverse.

are assembled from rearrangement of three noncontiguous gene segments, V, D, and J. Two-thirds of the time recombination between variable segments induces a frameshift in the open reading frame and the appearance of a premature termination codon. This results in the formation of considerable numbers of primary transcripts with out-of-frame $\mathrm{V}$ regions [8].

The germline, junctional, and combinatorial diversity, taken together with antigen-dependent changes such as hypermutation and receptor-editing with $B$ cell selection, can result in the formation of a large number of diverse $V$-gene sequences. The molecular identity of pathogen-specific $V$ genes in novel hybridomas is unknown and must be determined empirically via cloning and sequencing. The $V$-gene sequence is the primary sequence for encoding the antigen-binding-specific protein domain and is useful for studying $V$-gene immunogenetics. It is required for construction of the recombinant antibodies used for therapy and diagnostics, patent disclosure, and clone identity. Therefore, the nucleic acid sequence encoding the $V$ domain is of considerable importance [9]. Aberrant chains derived from the myeloma partner of hybridomas may dilute desired antibody sequences; these chains are the only ones binding the antigen in a pool of nonproductive antibody-like sequences $[7,9]$.

On the basis of the above information, we amplified the heavy chain variable region $(\mathrm{VH})$ and light chain variable region ( $\mathrm{V} L$ ) transcripts obtained from a hybridoma cell secreting anti-TfR mAb. Two chimeric antibodies were formed using functional $\mathrm{VL}$ and $\mathrm{VH}$ transcripts, and their antigen-binding properties were examined.

\section{Materials and Methods}

\subsection{PCR amplification and sequencing of murine $V L$ and $V H$ genes from hybridoma}

RNA was extracted from an anti-TfR mAb-secreting hybridoma using Trizol reagent (Invitrogen, Shanghai, China). cDNA was synthesized using a ReverTra Ace ${ }^{\circledR}$ kit (ToYoBo, Shanghai, China). The use of competitive $V$-gene PCR rendered amplification straightforward. Amplification primers VH-f1 to VH-f3 and VL-f1 to VL-f5 (Table 1) were designed to be complementary to the mRNA flanking $5^{\prime}$ end of the constant $\mathrm{C}_{\mathrm{H}}$ and $\mathrm{C} \kappa$ regions of mouse immunoglobulin, respectively. Primers VH-r1 and VL-r1 (Table 1) were designed to be complementary to the antisense cDNA encoding the conserved N-terminal region [10].

The PCR products were cloned into pGEM-T vector (Promega, Fitchburg, WI, USA) and transformed in Escherichia coli DH5 $\alpha$ cells. Transformed cells were cultured on solid Luria-Bertani medium containing $100 \mu \mathrm{g} / \mathrm{mL}$ ampicillin. cDNA sequencing was performed by the Invitrogen Company.

\subsection{Sequence analysis}

The ExPASy translation tool was used to translate DNA sequences to amino-acid sequences. The DNA sequences and amino-acid sequences were analyzed using IMGT/V-QUEST to identify functional $\mathrm{V} H$ and $\mathrm{V} L$ sequences and IMGT/DomainGapAlign to identify the V-J region within the functional $\mathrm{V} H$ and $\mathrm{V} L$ sequences [11]. Alignment of amino-acid sequences was conducted using the CLUSTAL W2 program from EBI. Prediction of the cleavage site and signal peptides was achieved using the 
TagetP 1.1 Server developed at the Technical University of Denmark (http://www.cbs.dtu.dk/services/TargetP/) and the SIG-Pred tool developed at the University of Leeds (http://bmbpcu36.leeds.ac.uk/prot analysis/Signal.html). A multinomial distribution model was used to determine whether the observed replacing mutations in any given gene segment occurred by chance [12] (http://www -stat.stanford.edu/immunoglobulin/).

\subsection{Analyzing origins of variable light chains NCBI/BLAST Blastn and blastp NIH programs} (http://blast.ncbi.nlm.nih.gov) were used to search for highly similar DNA and protein sequences against all obtained light chains separately. Because of the short length of the light chains obtained, the megablast program was used when running blastn analysis. The psi-blast program was used when running blastp analysis. Best matches were chosen by distance tree view of the alignment results. MEGA5.05 was used for building the phylogenetic tree.

\subsection{Molecular modeling and protein structure similarity analysis}

Three-dimensional (3D) structures modeling and loop refinement of corresponding proteins were analyzed using software Insight II and SWISS-MODEL. The 3D structures of chimeric single chain antibody fragment (scFv) were constructed by homology modeling based on immunoglobulin VL and VH domains containing highly similar amino-acid sequences and known structures in PDB [13]. SWISS-MODEL web-based programs were used for comparison of the derived sequences to identify the putative framework region (FR) and complementary determining region (CDR) loops in the variable genes of TfR mAbs. The template modeling Score (TM)-align program in the I-TASSER server was used to compare the predicted structure similarity of $\mathrm{V} \kappa 2$ and $\mathrm{V} \kappa 3$.

\subsection{Construction of chimeric anti-TfR antibody (chi-TfR)}

The mammalian expression vectors pFUSE-CHIg-hG1 (cat.code pfuse2-hchg1) and pFUSE2-CLIg-h $\kappa$ (cat.code pfuse2-hcl $\kappa$ ), each of which expresses human immunoglobulin constant regions, were purchased from InvivoGen (San Diego, CA, USA) and digested with restriction endonucleases. PCR products were subcloned into the above descended expression vectors to obtain $\mathrm{pV} \kappa$-CLIg and $\mathrm{pVH}$-CHIg, respectively. After linearization with NotI, recombinant $\mathrm{pV} \kappa$-CLIg and $\mathrm{pVH}$-CHIg were cotransfected into Chinese hamster ovary (CHO) cells using Lipofectamine 2000 (Invitrogen). The cells were cultured in 21 days, and the supernatants were harvested and analyzed by enzyme-linked immunosorbent assay (ELISA) to identify positive clones that produced human-mouse chimeric antibodies. Two positive clones were obtained, chi-C2 and chi-C3.

\subsection{ELISA}

A sandwich ELISA was designed to screen for clones producing antibodies containing human IgG1 and kappa $(\kappa)$ chain. Briefly, the supernatants from the transfectants were diluted serially from $1: 5$ to $1: 3,125$, and a suitable aliquoted $100 \mu \mathrm{L}$ of each supernatant was incubated at $4^{\circ} \mathrm{C}$ with "captured" antibody, goat anti-human $\kappa$ chain (Southern Biotechnology Associates, Birmingham, AL, USA), in wells overnight at $4^{\circ} \mathrm{C}$. After washing, $100 \mu \mathrm{L}$ of horseradish peroxidase-labeled goat anti-human immunoglobulin G (IgG) ( $\gamma$-chain specific; Southern Biotechnology Associates) was diluted to $1: 1,000$ and incubated at $37^{\circ} \mathrm{C}$ for $1 \mathrm{H}$. The optical density at $490 \mathrm{~nm}$ was determined with a (Genios Tecan, Austria) ELISA plate reader. All positive clones were retested to confirm a positive response.

\subsection{SDS-PAGE}

Samples were filtered through $0.45 \mathrm{~mm}$ filters and passed through a HiTrap Protein A HP (GE Healthcare, Sweden) as per the manufacturer's instructions. Chimeric antibody samples were analyzed by SDS-PAGE [4]. The gels were stained with Coomassie Brilliant Blue R 250. Gels were visualized using a gel image analysis system (Kodak Automatic Gel Imaging System, Shanghai, China), and the images archived as digital graphic files.

\subsection{Flow cytometry analysis}

The HL-60 (acute promyelocytic leukemia) cell was chosen as the target cell for high expression TfR; $1 \times 10^{6}$ cells were incubated at $4{ }^{\circ} \mathrm{C}$ for $1 \mathrm{H}$ with supernatants from transfectants expressing chi-C2 or chi-C3. The cells were washed $(3 \times)$ and incubated at $4 . \mathrm{C}$ for $30 \mathrm{Min}$ with fluorescein isothiocyanate (FITC)-conjugated goat anti-human IgG antibody and examined using a flow cytometer (Becton-Dickinson, San Jose, USA). In addition, HL-60 cells were incubated with parental murine TfR mAb. This treatment serves as a positive control. HL-60 cells were maintained in Roswell Park Memorial Institute (RPMI) 1640 medium, and nonspecific IgG cells were used as blank and negative controls, respectively.

\subsection{Statistical analysis}

Statistical significance of the data was calculated by analysis of variance or Student's $t$-test. A significance level of $P<0.05$ was chosen.

\section{Results}

\subsection{Sequence analysis}

\subsubsection{Functional sequences}

Six DNA sequences were obtained after sequencing: three sequences were amplified by $\mathrm{VH}$ primers, and three were amplified by VL primers. Using IMGT/V-QUEST server with a "mouse," one functional heavy-chain gene (VH1) and two functional light-chain genes $(V \kappa 2$ and $V \kappa 3)$ were obtained (Table 2). 
TABLE 2

Summary of IGMT analyses of obtained sequences

\begin{tabular}{|c|c|c|c|c|c|}
\hline Sequence name & Primer & $P / U N^{a}$ & $V$-gene and allele & J-gene and allele & IMGT analyses \\
\hline$V H 1$ & VH-f1 & $\mathrm{P}$ & IGHV1S56*01 (93.06\%) & IGHJ3*01 (91.67\%) & Productive IGH rearranged sequence \\
\hline VH2 & VH-f2 & UN & IGHV5-17*02F (86.11\%) & & No rearrangement found \\
\hline $\mathrm{V}_{\kappa} 1$ & VL-f1 & UN & $\mathrm{IG} \kappa \mathrm{V} 3-12 * 01$ (98.28\%) & $\mathrm{IG} \kappa \mathrm{J} 2 * 01(100.00 \%)$ & Unproductive $\mathrm{IG} \kappa$ rearranged sequence \\
\hline $\mathrm{V}_{\kappa} 2$ & VL-f2 & $\mathrm{P}$ & IG $\kappa$ V4-59*01 (98.19\%) & $\mathrm{IG} \kappa \mathrm{J} 2 * 03(96.97 \%)$ & Productive $\mathrm{IG} \kappa$ rearranged sequence \\
\hline$V_{\kappa} 3$ & VL-f3 & $\mathrm{P}$ & $\mathrm{IG} \kappa \mathrm{V} 6-17 * 01(100.00 \%)$ & $\mathrm{IG} \kappa \mathrm{J} 2 * 01(94.74 \%)$ & Productive $\mathrm{IG} \kappa$ rearranged sequence \\
\hline
\end{tabular}

aProductive (P), unproductive (UN).

Simnal oentide FR1 COR1 FR2 CDR2 FR3 COR3 FR4

$(-19-0) \quad(1-25)(26-33)(34-50)(51-58)(59-96)(97-105)(106-116)$

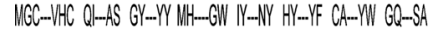

Sequences of heavy variable region

FIG. 1

Sequences of anti-TfR VH region.

\subsubsection{Cleavage site, FRs, and CDRs of VH1 sequence}

The cleavage site of functional $\mathrm{VH} 1$ sequence is between aminoacid residues 1 and 138 . When compared with the closest genes and alleles (Mus musculus IGHV1S56 ${ }^{*} 01$ and IGHJ3*01), three CDRs and four FRs are found in VH1 as determined by IGMT (Fig. 1).

\subsubsection{Cleavage site, FRs, CDRs, and alignment of $V_{\kappa} 2$ and $V_{\kappa} 3$ sequences}

Each of $\mathrm{V}_{\kappa} 2$ and $\mathrm{V} \kappa 3$ contains 183 amino acids, three CDRs, and four FRs. The cleavage site of $\mathrm{V}_{\kappa} 2$ is between positions 27 and position 28. The cleavage site of $\mathrm{V}_{\kappa} 3$ is between positions 25 and 26. Alignment of $\mathrm{V}_{\kappa} 2$ and $\mathrm{V} \kappa 3$ amino-acid sequences reveals that 79 amino acids (57\%) of the total amino-acid sequences are identical, whereas in $\mathrm{V}_{\kappa} 2$ the $\mathrm{V}$ segment and $\mathrm{J}$ segments are rearranged and lack an amino-acid residue between them. Two amino-acid residues occur between the $\mathrm{V}$ segment and $\mathrm{J}$ segment in $\mathrm{V} \kappa 3$ (Fig. 2).

\subsection{Somatic hypermutation and antigenic selection}

Statistical analysis was used to establish the types (e.g., silent or amino acid replacing) and distribution in the CDRs or FRs of somatic mutations. Twenty mutations were detected in the functional VH1 transcript (data not shown). As with the VL transcript, seven mutations were found in the $V_{\kappa} 2$ gene, one silent mutation in FR1, one nonsilent mutation in FR2, three nonsilent mutations in FR3, and two nonsilent mutations in CDR3. No somatic mutation was found in the $V_{\kappa} 3$ gene as predicted (Fig. 3). It strongly suggests that the $\mathrm{VH} 1$ and $\mathrm{V} \kappa 2$ transcripts were subjected to antigenic selection.

\subsection{Blast alignment of light chains}

Using PSI-BLAST searching on BLASTP suit/BLAST/NCBI with significant E-value $\left(<1 \mathrm{e}^{-40}\right)$ and high total score $(>140)$, more than 200 similar sequences were found for each light chain. According to the distance tree generated from the results provided by BLAST/NCBI, the $\mathrm{V}_{\kappa}$ protein sequence maps to an aberrant $\kappa$ transcript from mouse MOPC21 cell line (PIR:JL0073) with zero distance. The $\mathrm{V}_{\kappa} 3$ protein sequence is branched with $\kappa$-chain V-V region from MPC11 (SP:P01633.1), whereas the $\mathrm{V} \kappa 2$ protein sequence is branched with many single-chain antibodies (Table 3).

The $\mathrm{V} \kappa 1$ protein sequence exhibits $100 \%$ identity with the sequence PIR:JL0073. This result confirms that $\mathrm{V}_{\kappa} 1$ is the aberrant sequence arising from the myeloma partner MOPC21 of the hybridoma cell line. $\mathrm{V}_{\kappa} 2$ and $\mathrm{V} \kappa 3$ are new sequences. No identical sequence is yet to be found in the NCBI database.

\subsection{Phylogenetic trees}

Two phylogenetic trees have been built using light-chain DNA sequences and amino-acid sequences. Highly similar DNA sequences (2-3) from BLAST alignment results for each light chain were used to construct the phylogenetic tree (Fig. 4A). These results show that $\mathrm{V}_{\kappa} 1$ and $\mathrm{V} \kappa 3$ are closer than $\mathrm{V} \kappa 2$.

Because the amino-acid sequence of $\mathrm{V}_{\kappa} 3$ exhibits a greater homology to a $\kappa$ chain of MPC11, we used the protein sequences of MPC11 and MOPC21 and $\mathrm{V}_{\kappa} 2$ and $\mathrm{V}_{\kappa} 3$ to construct an amino acid phylogenetic tree (Fig. 4B). The results indicate that $\mathrm{V}_{\kappa} 3$ is more closely related to MPC11; each of them was grouped with MOPC21 but not $\mathrm{V} \kappa 2$.

Combining the two phylogenetic trees' results, we suggest that $\mathrm{V}_{\kappa} 3$ but not $\mathrm{V}_{\kappa} 2$ is a myeloma-derived light-chain sequence.

\subsection{D models of chimeric scFvs and protein structure comparison}

A 3D model of the variable domains showed that fragment, antigen binding region (Fab) of $\mathrm{TfR} \mathrm{mAb}$ had complete functional domains as well as binding grooves involved in the composition of the hydrophobic pocket (Fig. 5). 


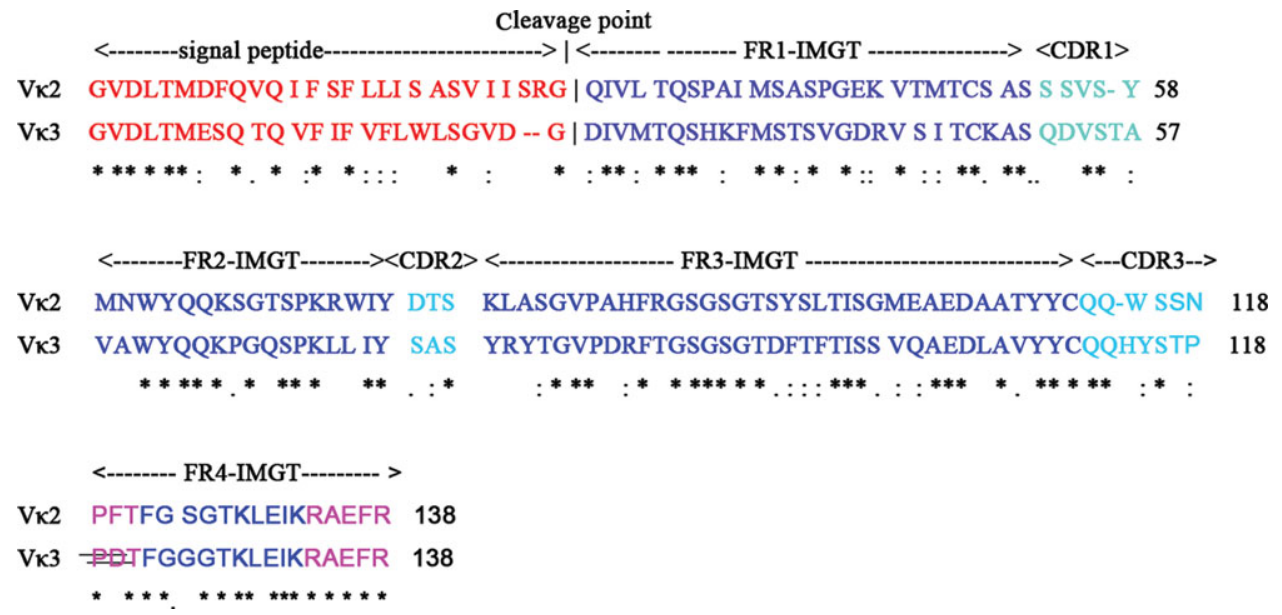

FIG. 2

Alignment of amino-acid sequences of anti-TfR $m A b V_{\kappa} 2$ transcript and $V_{\kappa} 3$ transcript. Pink highlight denotes signal peptide, blue highlight denotes $F R$, and yellow highlight denotes CDR. Red letters denote $J$ segment sequence.

Crossed-out letters denote residues between the $V$ and $J$ segments.

The TM these two models is 0.59 . These results strongly suggest that the two chimeric scFvs are nearly or fully identical.

\subsection{Expression and purification of chimeric antibody}

The supernatants of transfected CHO cells incubated for 5-7 days were collected and purified by binding to Protein A-sepharose. The purified products were diluted and detected by SDS-PAGE. Heavy and light chains of the expected molecular weights, 60 and $30 \mathrm{kDa}$, were observed under denaturing conditions (Fig. 6).

\subsection{Binding capability of chimeric antibody}

IgG of human origin was used as a standard to construct a standard curve to control to quantify chi-C2 and chi-C3. The concentration of chi-C2 and chi-C3 was calculated as 32 and $26 \mu \mathrm{g} / \mathrm{mL}$, respectively.

Flow cytometry (FCM) results reflect the binding strength by binding rate of the antibody-targeted recognition with TfRpositive tumor cells. The antigen-binding rate of parental murine mAb, chi-C2, and Chi-C3 was $69.68 \pm 13.47 \%$, $48.47 \pm 15.2 \%$, and $14.87 \pm 16.24 \%$, respectively. Each value is significantly higher than the negative control $(P<0.05)$.

The binding rate of chi-C3 $(14.87 \pm 16.24 \%)$ was significantly lower than those of chi-C2 and parental murine mAb $(P<0.05)$ (Fig. 7). No significant difference was found between parental murine $\mathrm{mAb}$ and chi-C2. This finding suggests that chi-C2 retains the antigen-binding ability of its corresponding parental $\mathrm{mAb}$. chi-C3 has a very weak antigen-binding ability, and it is significantly lower than chi-C2 and parental mAb.

\section{Discussion}

In these experiments, one B-cell-derived $\kappa$ transcript $(\mathrm{V} \kappa 2)$ and two myeloma-derived $\kappa$ transcripts $(\mathrm{V} \kappa 1$ and $\mathrm{V} \kappa 3)$ were amplified from one hybridoma that secreted anti-TfR mAb. One $\kappa$ transcript, $\mathrm{V} \kappa 1$, remained aberrant, but the other, $\mathrm{V} \kappa 3$,

\begin{tabular}{|c|c|c|c|c|}
\hline \multirow[b]{2}{*}{ Mutations } & \multicolumn{2}{|c|}{ 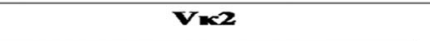 } & \multicolumn{2}{|c|}{ Vms 3} \\
\hline & $\mathbf{s}$ & $\mathbf{R}$ & $\mathbf{s}$ & $\mathbf{R}$ \\
\hline FRI & 1 & क & - & Ф \\
\hline CDRI & - & - & - & $\mathbf{0}$ \\
\hline FR2 & $\mathbf{0}$ & 1 & $\mathbf{0}$ & $\mathbf{0}$ \\
\hline CDR2 & $\mathbf{0}$ & $\mathbf{0}$ & $\mathbf{0}$ & $\mathbf{0}$ \\
\hline Fre3 & $\mathbf{0}$ & 3 & $\mathbf{0}$ & $\mathbf{0}$ \\
\hline Corz & $\mathbf{0}$ & 2 & $\mathbf{\Phi}$ & $\mathbf{0}$ \\
\hline FR sum & 1 & 4 & $\mathbf{0}$ & $\mathbf{0}$ \\
\hline CDR SUM & o & $\mathbf{2}$ & o & o \\
\hline $\boldsymbol{p}$ & $\mathrm{FR}=0.6652$ & $\mathrm{CDR}=0.6527$ & $\mathbf{F R}=\mathbf{0 . 0 0 0}$ & CDR $=1.000$ \\
\hline
\end{tabular}

Mutated IGV $V_{\kappa}$ sequences analyzed by

FIG. 3 http://www-stat.stanford.edu/immunoglobulin/. 
TABLE 3

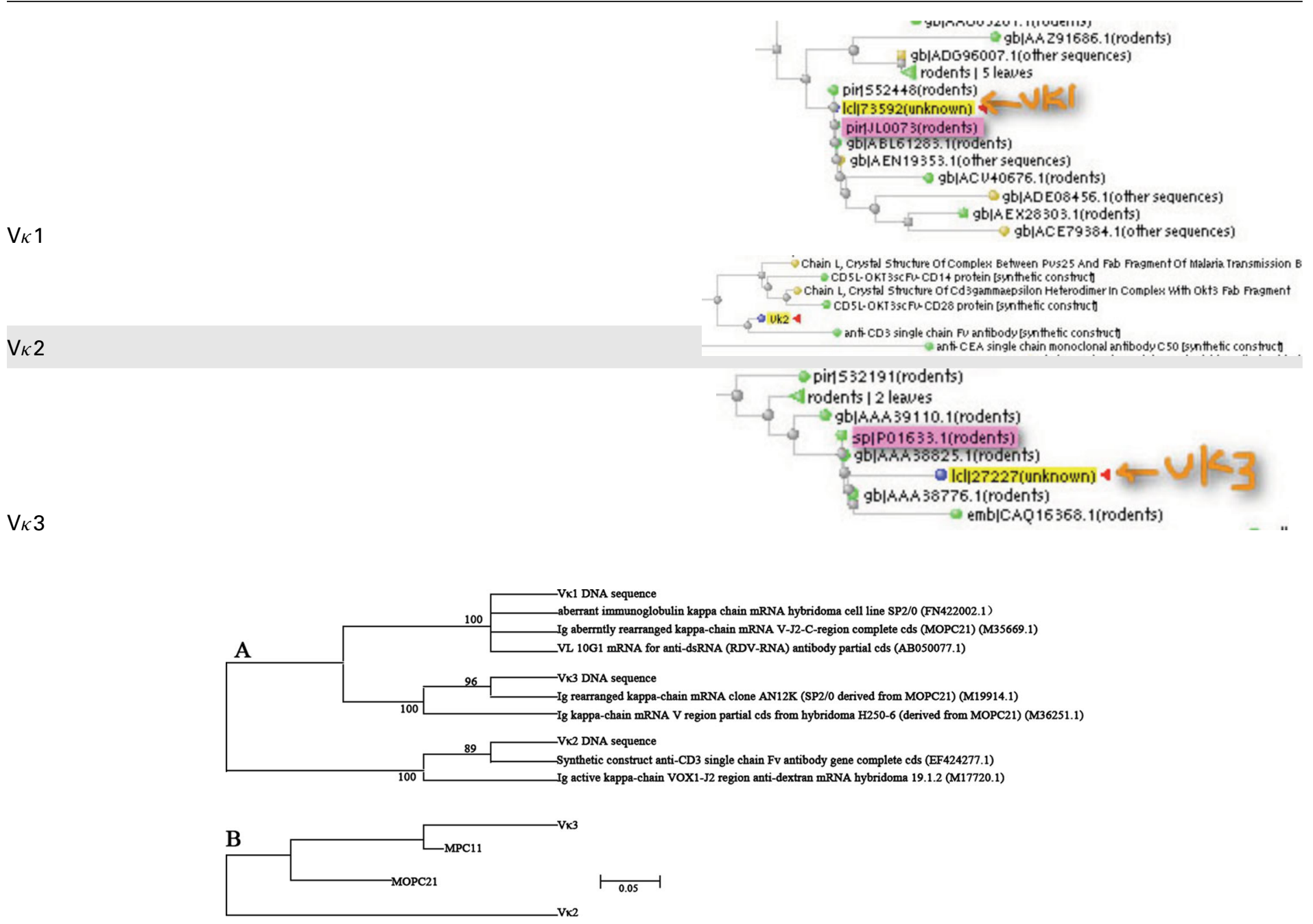

FIG. 4

(A) DNA sequence phylogenetic tree of light chains and $(B)$ amino-acid sequence phylogenetic tree of light chains.

contains a rearranged functional light chain. Many investigators, including ours, have amplified the myeloma-derived $\kappa$ by RT-PCR while attempting to clone the functional light chain from the B-cell fusion partner of hybridomas [9, 14-16].

Distinguishing the functional gene derived from the fused $\mathrm{B}$ cell and the myeloma-derived $\kappa$ transcript has been problematic. A hybrid approach has been used to analyze the three $\kappa$ chains, that is, we combined the "dry" bioinformatics evidence together with "wet" experimental evidence. Bioinformatics analyses provided the theoretical hypothesis that two $\kappa$ chains may derive from a myeloma ancestor. The antigen-binding experiment gave us the physical evidence to confirm the hypothesis inferred by informatics. IMGT analysis yielded the most reliable prediction in immune genes. To avoid bias and provide solid theoretical evidence, the sequence alignment was of importance. There are more than 15 million records in the Genebank $\mathrm{n} / \mathrm{r}$ database, and the number is growing daily. The short length of obtained $\kappa$ chains ( $\sim 130$ in terms of amino acid and $\sim 380 \mathrm{bp}$ in terms of their nucleotide sequence), using a normal blast algorithm allowed us to find more than 150 similar sequences (e.g., using blastn retrieved 170 sequences similar to the $\mathrm{V} \kappa 3$ nucleotide sequence). It is difficult to characterize light-chain sequences using this approach. Therefore, in these studies, we applied Mega-BLAST for determining nucleotide sequences, PSI-BLAST for amino-acid sequences, and combined the Blast tree view to select the most similar sequences from the results ( $\sim 120$ sequences). This allowed filtering out one-third of the total results using the normal BLAST program. Phylogenetic trees built from amino-acid sequences and nucleotide sequences support the hypothesis that $\mathrm{V}_{\kappa} 3$ and $\mathrm{V}_{\kappa} 1$ 


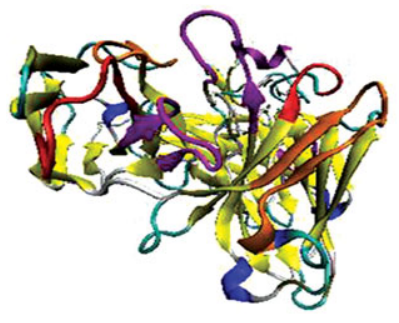

A

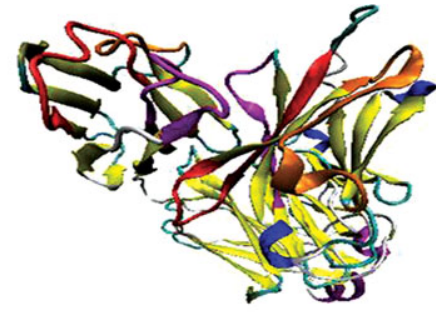

$\mathbf{B}$
FIG. 5

Computer-assisted modeling of Fab of TfR $m A b$ (A: chi-C2 Fab region and B: chi-C3 Fab region). $A$ model of anti-TfR $\mathrm{VH}$ and $V_{\kappa}$ is shown as blue backbone. The $\beta$-layer secondary structures of $\mathrm{VH}$, $V L$, and CDR1-3 are depicted in yellow. are of the same myeloma origin and they are closer to MPC11 or MOPC21. In addition, multiple mutations are predicted in $\mathrm{V}_{\kappa} 2$ but not in $\mathrm{V}_{\kappa} 3$. The results support the hypothesis that $\mathrm{V}_{\kappa} 2$, but not $\mathrm{V}_{\kappa} 3$, was subjected to antigen selection.

Why does the transcript $\mathrm{V} \kappa 3$ exist in the hybridoma that secreted anti-TfR mAb, and why is its origin much closer to MPC11? First, most mouse myeloma cell lines available for fusions are descendants of either MPC11 BALB/c myeloma or MOPC21 BALB/c myeloma lines [16]. The anti-TfR mAbsecreting hybridoma cell line's myeloma partner may come from a fusion of MPC11 and MOPC21. Brady et al. found two MOPC-derived $\kappa$ transcripts in their murine MOPC21-derived hybridomas. One is identical to the full-length $\kappa$ chain of MPC11, and the other is the MPC11 aberrant $\kappa$ transcript, which has no variable region [17]. Second, hybridomas are stable in culture and continue to produce the same antibody indefinitely. However, rare mutant clones, which produce an antibody that has changed its specificity, affinity, class, or subclass, can arise [18]. The existence of transcript $V_{\kappa} 3$ may be due to the mutation or contamination of the hybridoma cell line. Last, although two functional $\kappa$ transcripts have been amplified, the mentioned cell line secretes only one type of mAb. It seems possible that the two light chains exist at the transcript level but only one light chain is secreted in our hybridoma. For example, in the case of hybridoma CEA 66-E3, only one $\mathrm{Ab}$ is secreted, although it contains significant levels of multiple Ig transcripts.

The major problem in rapidly obtaining chain variable genes from a hybridoma is the occurrence of aberrant mRNAs that can be transcribed but that have no function. If the aberrant gene is used in the construction of the chimeric or humanized antibody, it may produce immunoglobulin that has low affinity for the antigen and is completely nonfunctional [19]. We encountered such a situation in our experiments.

Although structure simulation shows that chi-C2 and chiC3 are very similar in the protein structure level, their actual affinity functions are different. This could be attributed to the different origins of $\mathrm{V} \kappa 2$ and $\mathrm{V} \kappa 3$. However, when compared

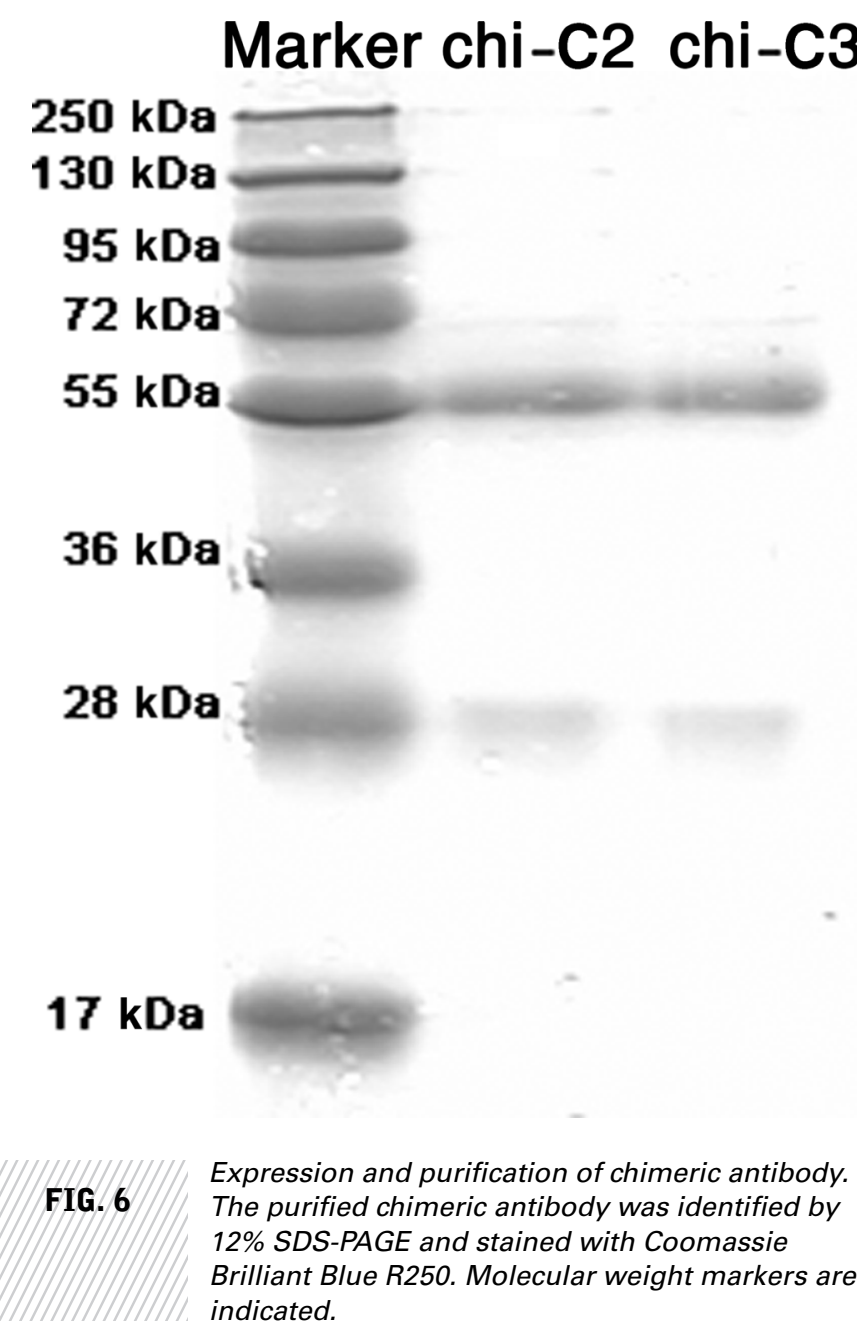

with the negative control, chi-C3 still possesses a weak affinity, which is significantly lower than chi-C2. Because chi-C3 was constructed with functional $\mathrm{VH}$ and $\mathrm{V} \kappa 3$, the functional $\mathrm{VH} 1$ chain may responsible for the weak affinity of chi-C3.

Several strategies may decrease the amplification of aberrant variable genes, but they have limitations primarily because the aberrant genes may be highly homologous to the functional ones. As a result, the more information we have about the aberrant genes, the higher efficiency of functional gene amplification we can obtain and the better we can avoid the aberrant genes in antibody engineering.

In our experiment, two aberrant $V H$ genes may arise from an unproductive transcript in myeloma cells, a rearranged transcript in B cells, or a gene mutation in a fusion of the two kinds of cells. After the analysis, we found the two unproductive heavy chains were also very prevalent in the study of hybridoma, and found that it has a high similarity with Ig heavychain V region MOPC21, a previously described aberrantly rearranged heavy-chain gene (GenBank accession no. X58634) believed to be derived from the fusion partner P3×63-Ag8.653. Aberrant $V H$ genes are less often reported but are more 


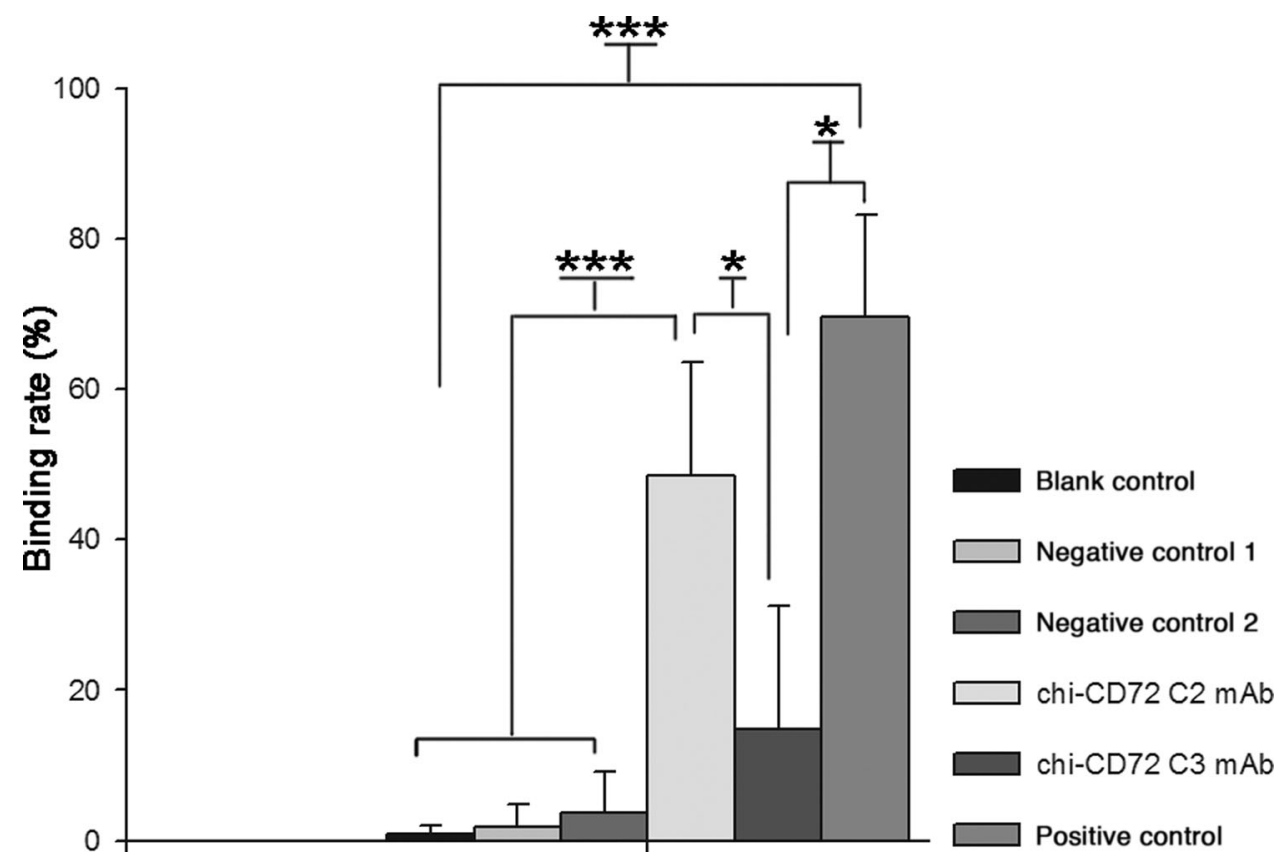

FIG. 7

The antigen-binding ability of chi-TfR Ab to the TfR expressed on HL-60 cells. Data were represented as the means $\pm S D$ of three independent experiments. A significance level of $P<0.05$ was chosen. ${ }^{*} P<0.05,{ }^{*} P<0.01$, and ${ }^{*}{ }^{*} P<0.001$. complicated and not as widely recognized as that of the MOPC21 $\kappa$ allele $[19,20]$.

More than two different variable genes in one hybridoma were rare. At first, we did not place any significance on aberrantly $V H$ genes, and two functional $\mathrm{V}_{\kappa}$ chains stimulated our interest. The sequence alignment, phylogenetic tree, somatic hypermutation prediction, and 3D-molecular structure modeling were used to predict the origin of the two $\kappa$-chain transcripts. The experimental evidence of chi-C2 and chi-C3 in accordance with bioinformatics evidence showed that $\mathrm{V}_{\kappa} 3$ is derived from the myeloma partner of the hybridoma; and the $\mathrm{V} \kappa 2$ is derived from the $\mathrm{B}$ cell. These results encouraged us to analyze the sources of two aberrantly $V H$ genes, the distinct innate MOPC21H allele genes gave us more evidence to support our result. Another attempt is to find simple and effective methods to identify functional and aberrant genes. We did a restriction-enzyme polymorphism analysis on aberrant VL gene $\left(V_{\kappa} 1\right)$, functional gene $\left(V_{\kappa} 2\right)$, and the myeloma-likelyderived $V_{\kappa} 3$ gene. The results show that $V_{\kappa} 1$ has $B c i \mathrm{VI}$ site, but not $\mathrm{V}_{\kappa} 2$ and $\mathrm{V}_{\kappa} 3$. Only $\mathrm{V}_{\kappa} 2$ has many restriction-enzyme sites, such as BamHI, HindIII, EcoRI, KpnI, and $X b a \mathrm{I}$, but not $\mathrm{V} \kappa 1$ and $\mathrm{V} \kappa 3$. The same analysis was done with nonfunctional heavy chains. We found that two nonfunctional heavy chains have 62 identical restriction-enzyme sites, and then based on these, we chose every two $\mathrm{VH}$ chains from 16 families of VH1-16 of mouse IgH V germline sequences, and found these families and two aberrant $\mathrm{VH}$ have many identical restriction-enzyme sites. These results showed that enzyme sites, such as BsmBI, Esp3I
DraII, PpuMI, Psp5II, and Eco0109I, may be used to distinguish the innate $\mathrm{VH}$ of MOPC21.

In addition, some investigators suggest that if a hybridoma expresses a variety of variable region genes, only one advantage light-chain and heavy-chain antibody genes are fully functional, whereas others will lose their functionality [17]. This situation may explain the phenomenon reported in this article.

\section{Conclusions}

In this study, we report that two distinct functional $\kappa$-chain transcripts were present in all the transcripts amplified from a hybridoma that secretes anti-TfR mAb. Coexistence of distinct light transcripts from a single hybridoma is not uncommon. A few previous studies have demonstrated the coexistence of functional $\kappa$-chain transcripts. However, when using functional $\kappa$-chain transcripts to construct chimeric mAbs, apparently only one light transcript is involved in the formation of a functional chimeric mAb that has an affinity similar to the mAb secreted from the hybridoma. In-depth combined bioinformatics analysis based on sequence information is needed before constructing a mAb. The approach used in this study establishes a standard workflow for constructing mAbs in our laboratory, as well as a reference workflow for any other antibody engineering laboratories. The mAb was amplified using degenerate primers, and the primers more frequently amplified were analyzed for nonfunctional sequences. For example, primers $\mathrm{V}_{\kappa} 1, \mathrm{~V} H 2$, and VH3 are reliable for obtaining aberrant chains; the primer sequences show some continuous sequences are identical with endogenous genes of MOPC21. We deleted these primers and subsequent amplification was relatively easy. Finally, it is evident that at the primer design stage, the primers amplifying the $\mathrm{V} H 2, \mathrm{~V} H 3, \mathrm{~V} \kappa 1$, and $\mathrm{V} \kappa 3$ sequences should be filtered out. 


\section{Acknowledgements}

This work was supported in part by the 863 Program of China (2012AA02A307 to GX.S.).

\section{References}

[1] Daniels, T. R., Delgado, T., Helguera, G., and Penichet, M. L. (2006) Clin. Immunol. 121, 159-176.

[2] Daniels, T. R., Ortiz-Sanchez, E., Luria-Perez, R., Quintero, R., Helguera, G., Bonavida, B., Martínez-, O., and Penichet, M. L. (2011) J. Immunother. 34, 500-508.

[3] Peng, J. L., Wu, S., Zhao, X. P., Wang, M., Li, W. H., Shen, X., Liu, J., Lei, P., Zhu, H. F., and Shen, G. X. (2007) Biochem. Biophys. Res. Commun. 354, 864-871.

[4] Shen, X., Hu, G. B., Jiang, S. J., He, F. R., Xing, W., Li, L., Yang, J., Zhu, H. F., Lei, P., and Shen, G. X. (2009) Protein Eng. Des. Sel. 22, 723-731.

[5] Shen, X., Zhu, H. F., He, F. R., Xing, W., Li, L., Liu, J., Yang, J., Pan, X. F., Lei, P., Wang, Z. H., Shen, G. X. (2008) Int. Immunopharmacol. 8, 1813-1820.

[6] Hong, Y., Yang, J., Shen, X., Zhu, H., Sun, X., Wen, X., Bian, J., Hu, H., Yuan, L., Tao, J., Lei, P., Shen, G. (2013) Cancer Immunol. Immunother. 62, 447-454.

[7] Krebber, A., Bornhauser, S., Burmester, J., Honegger, A., Willuda, J., Bosshard, H. R., and Plückthun, A. (1997) J. Immunol. Methods 201, 3555.
[8] Chemin, G., Tinguely, A., Sirac, C., Lechouane, F., Duchez, S., Cogné, M., and Delpy, L. (2010) J. Immunol. 184, 5009-5017.

[9] Yuan, X., Gubbins, M. J., Berry, J. D. (2004) J. Immunol. Methods 294, 199-207.

[10] Zhou, C., Shao, J., Wang, X., Zhu, H., Zhang, Y., Xiong, W., and Shen, G. (1998) Hybridoma 17, 275-282.

[11] Brochet, X., Lefranc, M. P., and Giudicelli, V. (2008) Nucleic Acids Res. 36, 503-508.

[12] Lossos, I. S., Tibshirani, R., Narasimhan, B., and Levy, R. (2000) J. Immunol. $165,5122-5126$.

[13] Barderas, R., Desmet, J., Timmerman, P., Meloen, R., and Casal, J. I. (2008) Proc. Natl. Acad. Sci. USA 105, 9029-9034.

[14] Altenburger, W., Steinmetz, M., and Zachau, H. G. (1980) Nature 287, 603607.

[15] Carroll, W. L., Mendel, E., and Levy, S. (1998) Mol. Immunol. 25, 991-995.

[16] Bartal, A. H., and Hirshaut, Y. (1987) Methods of Hybridoma Formation. p. 11, Humana Press, New York, NY.

[17] Brady, J. L., Corbett, A. J., McKenzie, B. S., and Lew, A. M. (2006) J. Immunol. Methods 315, 61-67.

[18] Pirofski, L., Casadevall, A., Rodriguez, L., Zuckier, L. S., and Scharff, M. D. (1990) J. Clin. Immunol. 10(6 Suppl), 5S-14S.

[19] Ding, G., Chen, X., Zhu, J., and Cao, B. (2010) Cell. Mol. Immunol. 7, 349354.

[20] Irani, Y., Tea, M., Tilton, R. G., Coster, D. J., Williams, K. A., and Brereton, H. M. (2008) J. Immunol. Methods 336, 246-250. 